\title{
Protein tau concentration in blood increases after SCUBA diving: an observational study
}

\author{
Anders Rosén ${ }^{1,2}\left(\mathbb{D}^{-} \cdot\right.$ Mikael Gennser $^{3} \cdot$ Nicklas Oscarsson $^{1,2}\left(\mathbb{D} \cdot\right.$ Andreas Kvarnström $^{1,2} \cdot$ Göran Sandström $^{2,4}$. \\ Helen Seeman-Lodding ${ }^{1,2} \cdot$ Joel Simrén ${ }^{5,6}$ (1) Henrik Zetterberg ${ }^{5,6,7,8,9}$ (i)
}

Received: 22 September 2021 / Accepted: 5 January 2022 / Published online: 10 February 2022

(c) The Author(s) 2022

\begin{abstract}
Purpose It is speculated that diving might be harmful to the nervous system. The aim of this study was to determine if established markers of neuronal injury were increased in the blood after diving.

Methods Thirty-two divers performed two identical dives, $48 \mathrm{~h}$ apart, in a water-filled hyperbaric chamber pressurized to an equivalent of $42 \mathrm{~m}$ of sea water for $10 \mathrm{~min}$. After one of the two dives, normobaric oxygen was breathed for $30 \mathrm{~min}$, with air breathed after the other. Blood samples were obtained before and at 30-45 and $120 \mathrm{~min}$ after diving. Concentrations of glial fibrillary acidic, neurofilament light, and tau proteins were measured using single molecule array technology. Doppler ultrasound was used to detect venous gas emboli.

Results Tau was significantly increased at 30-45 min after the second dive $(p<0.0098)$ and at 120 min after both dives $(p<0.0008 / p<0.0041)$. Comparison of matching samples showed that oxygen breathing after diving did not influence tau results. There was no correlation between tau concentrations and the presence of venous gas emboli. Glial fibrillary acidic protein was decreased 30-45 min after the first dive but at no other point. Neurofilament light concentrations did not change. Conclusions Tau seems to be a promising marker of dive-related neuronal stress, which is independent of the presence of venous gas emboli. Future studies could validate these results and determine if there is a quantitative relationship between dive exposure and change in tau blood concentration.
\end{abstract}

Keywords Biomarkers $\cdot$ Brain $\cdot$ Central nervous system $\cdot$ Diving $\cdot$ Diving research $\cdot$ Proteins $\cdot$ Venous gas embolism

\section{Abbreviations}

BMI Body mass index

CNS Central nervous system

$\mathrm{CV}$ Coefficient of variation

Communicated by Guido Ferretti.

Anders Rosén

anders.rosen@vgregion.se

1 Department of Anaesthesia and Intensive Care Medicine, Sahlgrenska University Hospital, Gothenburg, Sweden

2 Department of Anaesthesia and Intensive Care Medicine, Institute of Clinical Sciences, Sahlgrenska Academy, University of Gothenburg, Gothenburg, Sweden

3 Swedish Aerospace Physiology Centre, Division of Environmental Physiology, Department of Biomedical Engineering and Health Systems, School of Engineering Sciences in Chemistry, Biotechnology and Health, Royal Institute of Technology, KTH, Stockholm, Sweden

4 Swedish Armed Forces, Center for Defence Medicine, Gothenburg, Sweden
DCS Decompression sickness

GFAp Glial fibrillary acidic protein

KISS Kisman integrated severity score

KM Kisman Masurel

$\mathrm{kPa} \quad$ Kilopascal
5 Clinical Neurochemistry Laboratory, Sahlgrenska University Hospital, Gothenburg, Sweden

6 Department of Psychiatry and Neurochemistry, Sahlgrenska Academy, University of Gothenburg, Mölndal, Sweden

7 Department of Neurodegenerative Disease, Institute of Neurology, University College London, London, UK

8 UK Dementia Research Institute, University College London, London, UK

9 Hong Kong Center for Neurodegenerative Diseases, Hong Kong, China 
msw Meters of seawater

NfL Neurofilament light

Simoa Single molecule array

SwAF Swedish armed forces

tau Protein tau

UCH-L1 Ubiquitin carboxy-terminal hydrolase L-1

VGE Venous gas emboli

QC Quality control

\section{Introduction}

It is well-known that diving is not without certain risks. The diver is exposed to increased ambient pressure that causes inert gas accumulation and inhaled gases can, at sufficient depth, exert a noxious effect on the central nervous system (CNS). Oxygen becomes harmful to the CNS when its partial pressure exceeds $160 \mathrm{kPa}$, equivalent to a depth of about $66 \mathrm{~m}$ of seawater (msw) when breathing air. Symptoms of oxygen toxicity range from cognitive and sensory impairment to manifest convulsions (Bitterman 2004). Adverse effects of nitrogen, typically behavioural and intellectual disturbances, become apparent as the partial pressure of nitrogen exceeds $300 \mathrm{kPa}$ (equivalent to a depth of about $28 \mathrm{msw}$ when breathing air) and gradually worsen with increasing pressure (Clarke 2015).

Rapid ascent at the end of a dive may cause barotrauma to the lungs and sinuses. Nitrogen taken up by the body during diving may come out of solution as ambient pressure decreases and form gaseous bubbles in both blood and tissues, which is a common occurrence after diving (Nishi 1981, Eckenhoff 1990). Nitrogen bubbles in the blood can be detected by Doppler ultrasound and are referred to as venous gas emboli (VGE) (Blogg et al. 2014). The amount of VGE in the blood after diving depends on both the amount of accumulated inert gas and decompression stress, but a substantial individual variability exists (Germonpre and Balestra 2017, Papadopoulou et al. 2018). Though VGE is a normal phenomenon after diving, release of dissolved gas as bubbles is considered to be the cause of decompression sickness (DCS) (Eftedal et al. 2007, Blogg et al. 2014). The risk of DCS is correlated to VGE load after diving (Sawatzky 1991, Eftedal et al. 2007). Oxygen breathing employed before (Castagna et al. 2009, Bosco et al. 2010), during (Bosco et al. 2010) and after diving (Blatteau and Pontier 2009) have all been associated with reduced amounts of inert gas bubbles (VGE) in blood, as have active hydration (Gempp et al. 2008), whole body vibration (Germonpre et al. 2010) and sauna-induced heat exposure (Gempp and Blatteau 2010) before diving.

Historically, there have been discussions as to whether repeated, and especially deep diving could be harmful to the nervous system. Several radiological studies involving divers have been published, but the results yielded are hard to interpret, as both the cause and clinical significance of observed CNS lesions often remain uncertain (Knauth et al. 1997, Tetzlaff et al. 1999, Grønning and Aarli 2011, Kohshi et al. 2014, Coco et al. 2019). There have been reports of impaired cognitive performance among both professional and recreational divers without documented DCS, but results from published studies are conflicting. Neuropsychological changes were found in $20 \%$ of saturation divers when tested before and after 3.5 years of diving (Vaernes et al. 1989). Saturation divers with self-reported forgetfulness and loss of concentration were found to have mild cognitive deficits when tested objectively and compared to matched controls (Taylor et al. 2006). In a retrospective study, experienced saturation divers did not only have more neurological symptoms but also exhibited more subjective problems with memory and concentration than individuals in a nondiving control group (Todnem et al. 1990, 1991) and experienced recreational divers without a history of DCS have been shown to have a worse short-term memory compared to non-diving control subjects when tested after an average of 12 years diving (Hemelryck et al. 2014). Studies have found inferior neuropsychometric test results among recreational (Balestra et al. 2016), professional (Ergen et al. 2017) and experienced breath-hold divers (Billaut et al. 2018) compared to non-diving controls. Depth and number of dives have been reported to have a negative influence on cognitive performance among recreational divers when tested retrospectively (Slosman et al. 2004). However, contrary to these results, one retrospective study found no difference in neuropsychometric test results when professional divers were compared to matched controls (Cordes et al. 2000), nor was there evidence of neuropsychological impairment among professional, non-saturation divers without a history of DCS when followed-up over 12 years in a longitudinal study (Bast-Pettersen et al. 2015). Post-dive cognitive function studies such as these are hard to interpret, as confounding factors might influence the results, and decreased neuropsychological test performance is not tantamount to neurological impairment.

Increased partial pressure of oxygen (Ferrer et al. 2007, Camporesi Bosco 2014, Bosco et al. 2018) and nitrogen (Bhullar et al. 2016), present during diving, could cause oxidative stress and increased levels of reactive oxygen species (ROS), which could potentially harm cells in the CNS. If diving has a detrimental effect on the brain, it would likely induce changes in the concentration of biochemical markers known to increase in response to CNS trauma or neuronal stress. GFAp is an astrocytic protein involved in several neuronal processes, including synaptic transmission. It has been studied both as a marker of neuronal damage in the context of traumatic brain injury and cerebral haemorrhage when venous concentration increases (Foerch et al. 
2012, Zetterberg and Blennow 2016, Gill et al. 2018), and as a marker of degenerative disease (Siracusa et al. 2019). Animal studies suggest that increased immunologic and neuronal activity, as well as neuronal stress could result in changed concentrations of GFAp (Wang and Hatton 2009, Brenner 2014, Femenia et al. 2018). Neurofilament light $(\mathrm{NfL})$ is a neuronal cytoskeletal component found mainly in myelinated subcortical axons. Serum NfL concentrations are increased in patients with cerebral traumas, ranging from sports related concussions (Shahim et al. 2018) to severe traumatic brain injuries (Zetterberg and Blennow 2016, Shahim et al. 2016). Neurodegenerative disorders such as multiple sclerosis and Alzheimer's disease are associated with increased NfL concentrations in blood (Bergman et al. 2016, Kahlil et al. 2018). Uncomplicated general anaesthesia in conjunction with orthopedic surgery has also been associated with increased blood levels of NfL (Evered et al. 2018).

Tau is a microtubular protein present mainly in unmyelinated cortical axons but also, to a lesser extent, in the liver, kidneys and testes. Tau could be both passively released as a result of manifest axonal damage (Zetterberg and Blennow 2016) and actively secreted in connection to increased neuronal activity in response to stress (Sato et al. 2018). Neurodegenerative diseases, sports related concussions, boxing and traumatic brain injuries are all associated with increased tau levels in blood (Zetterberg et al. 2006, Neselius et al. 2012, Zetterberg and Blennow 2016, Mattson 2017, Shahim et al. 2018). As with NfL, increased blood concentrations of tau have been found after uneventful general anaesthesia in conjunction with orthopedic surgery (Evered et al. 2018). Physiological stressors such as protracted apnea among breath-hold divers (Gren et al. 2016) and high intensity interval training (Battista et al. 2018) have both been associated with increased tau levels in blood. A small pilot study found increased serum tau levels in blood after repeated deep, open sea diving (Rosén et al. 2019). In contrast, tau levels were not increased in cerebrospinal fluid in a small study on divers with DCS, though only one of seven patients in the study had CNS symptoms (Shahim et al. 2015). In addition, serum tau did not increase in response to nitrox saturation exposure in a study on submariners (Rosén et al. 2020).

The present study tested the hypothesis that diving to $42 \mathrm{msw}$ would, via neuronal stress, incur a change in GFAp, $\mathrm{NfL}$ and tau. It was also investigated whether breathing normobaric oxygen after diving changed the amount of GFAp, $\mathrm{NfL}$ and tau in blood.

\section{Materials and methods}

The study was conducted at the Swedish Armed Forces (SwAF) naval base in Karlskrona, Sweden, during June 2015 and November 2018, as part of a project where subjects performed identical dives breathing either air or normobaric oxygen after decompression. The study was prospective, observational, registered at ClinicalTrials.gov (NCT02468752) and approved by Swedish ethical review authorities. Participants were recruited among professional divers from the SwAF, the Swedish Coast Guard and the Swedish Police. Written consent was obtained from all subjects before the start of the study.

Altogether, 33 professional divers were recruited. Data concerning age, gender, weight, height, medication and prior DCS were collected from all subjects. A water-filled hyperbaric chamber was used to simulate diving. After entering the chamber, the study subjects immersed themselves in water and rested in a horizontal position close to the bottom of the chamber for the duration of the experiment. All study subjects breathed air using an open circuit system.

The chamber was electronically pressurized to $521 \mathrm{kPa}$ (equivalent of $42 \mathrm{msw}$ ) for $10 \mathrm{~min}$ by an external operator. Compression speed was $200 \mathrm{kPa} / \mathrm{min}$, and decompression speed $90 \mathrm{kPa} / \mathrm{min}$ (equivalent to $9 \mathrm{msw} / \mathrm{min}$ ), with a safety stop made at $151 \mathrm{kPa}$ (equivalent to $5 \mathrm{msw}$ ) for $3 \mathrm{~min}$. After surfacing, either oxygen or air was breathed for $30 \mathrm{~min}$ using tight-fitting face masks with demand valves. The breathing gas a particular study subject used after the first dive was randomly chosen by an independent operator and hence unknown to both study subjects and study personnel. In June 2015 (16 subjects, 32 dives), oxygen or air breathing commenced immediately after surfacing, while in November 2018 (16 subjects, 32 dives), mask breathing was deliberately delayed by $15 \mathrm{~min}$. After a predetermined interval of $48 \mathrm{~h}$, all subjects performed an identical second dive, with a switch of the breathing gases afterwards. Study subjects and all study personnel were unaware of the breathing gas used after a particular dive throughout the trial.

Immediately after surfacing, the subjects were monitored for VGE by a blinded operator using precordial Doppler ultrasound (DBM9008, Techno Scientific Inc, Ontario, Canada). Measurements were made every five minutes during the first $30 \mathrm{~min}$ and every $15 \mathrm{~min}$ thereafter for a further $90 \mathrm{~min}$. Venous gas emboli were measured using the Kisman Masurel (KM) grading system, which is an ordinal scale based on categorical data describing amplitude, frequency and duration of VGE (Kisman 1978a). Maximal $\mathrm{KM}$ grades were recorded for every subject after each dive $\left(\mathrm{VGE}_{\max }\right)$. For each subject, the Kisman Integrated Severity Score (KISS) algorithm (Kisman et al. 1978b, Jankowski et al. 2004) was used to convert all KM grades collected 
from 0-30 min and 0-120 min after diving into individual integrated scores (KISS 30 min and KISS $_{120 \mathrm{~min}}$ ).

Venous blood samples were collected 1-3 h before the first dive (sample 1, baseline), after mask breathing had ended (sample 2, 30-45 min after diving) and $120 \mathrm{~min}$ after diving (sample 3 ). For the second dive, the same blood protocol was performed, with the resulting samples named 4-6. Besides providing a baseline value before the second dive, Sample 4 was also used as the last ( $48 \mathrm{~h}$ after diving) in the series of samples taken after the first dive.

Plasma EDTA tubes (Vacuette nr 454410, Hettish Labinstrument AB, Sweden) were used for blood collection and samples were centrifuged for $15 \mathrm{~min}$ at $2500 \mathrm{rpm}$ and $4^{\circ}$ centigrade (Sorvall ST 8/8R centrifuge, Thermo Scientific, Germany). Directly after centrifugation, aliquots of $500 \mu \mathrm{L}$ plasma were frozen at $-18^{\circ}$ centigrade for $1-4$ days, transported on dry ice and then stored at $-78^{\circ}$ centigrade until analysed.

GFAp, NfL and tau concentrations were measured using the Human Neurology 4-Plex A assay on an HD-1 Single molecule array (Simoa) instrument (Quanterix, Lexington, MA, USA). The 4-Plex assay also included ubiquitin carboxy-terminal hydrolase L-1 (UCH-L1), which was not further assessed in this study. All samples were analysed together in one batch. For quality control (QC) samples with GFAp concentrations of $87.2 \mathrm{pg} \mathrm{mL}^{-1}$ and $465.2 \mathrm{pg} \mathrm{mL}^{-1}$, coefficients of variation (CVs) were $7.3 \%$ and $3.2 \%$ respectively, for QC samples with $\mathrm{NfL}$ concentrations of $8.7 \mathrm{pg} \mathrm{mL}^{-1}$ and $46.2 \mathrm{pg} \mathrm{mL}^{-1} \mathrm{CVs}$ were $9.2 \%$ and $3.7 \%$ and for quality control (QC) samples with tau concentrations of $1.0 \mathrm{pg} \mathrm{mL}^{-1}$ and $2.6 \mathrm{pg} \mathrm{mL}^{-1} \mathrm{CVs}$ were $4.3 \%$ and $7.9 \%$, respectively.

\section{Statistics}

Compilation of demographic data was performed using IBM SPSS ${ }^{\circledR}$ v24 (IBM, Armonk, NY, USA). Statistical analyses regarding absolute changes in GFAp, NfL and tau were performed by an independent statistical company using SAS® v9.4 (Cary, NC, USA). Concentrations of GFAp, NfL and tau $\left(\mathrm{pg} \cdot \mathrm{mL}^{-1}\right)$ were presented with both mean and median values with standard deviation (SD) and range (minimum and maximum values) stated. Fisher's non-parametric permutation test for paired observations was used in analyses within groups and Fisher's non-parametric permutation test in analyses between groups. Mean difference with $95 \%$ confidence interval (CI) was considered the main result. All significance tests were two-sided with a significance level of $5 \%$. Spearman's rank correlation test was used to assess if there were any correlations between GFAp, NfL or tau concentrations and the presence of VGE. A positive or negative Spearman correlation coefficient greater than 0.8 was used as a limit to accept correlation between variables. KISS values and relative changes in GFAp, NfL and tau (\%) were computed using Microsoft $\odot$ Office Excel 2018 (Microsoft Corporation, Redmond WA, USA).

\section{Missing data}

One subject from the June 2015 cohort was excluded from the study due to dysbarism at the start of his first dive. His demographic data and Sample 1 results were omitted from all compilations and analyses. All remaining 32 divers completed two dives. There were no missing data for GFAp, $\mathrm{NfL}$ and tau. All VGE data were collected according to the protocol.

\section{Results}

\section{Demographics}

Among the 32 subjects that completed the study, 31 were males and one was female. Mean age was 37.9 years [standard deviation (SD) 8.1, range 26-55 years) and mean body mass index (BMI) was 25.4 (SD 1.7, range 21.4-29.2). Two subjects had experienced shoulder pain, possibly a sign of decompression sickness (DCS), earlier in their diving career but none had been treated in a hyperbaric oxygen chamber. One subject was prescribed antihypertensive medication with losartan, another for unknown reasons used diclofenac once between the two dives.

\section{Biomarkers of neuronal injury}

\section{Effect of breathing oxygen after diving}

To assess if breathing oxygen after diving had an effect on GFAp, NfL or tau concentrations, samples obtained after breathing oxygen (samples 2 and 3 or 5 and 6) were compared to matching samples after breathing air (samples 5 and 6 or 2 and 3, respectively). A potential period effect was adjusted for by using Fisher's non-parametric test to analyse changes in GFAp, NfL and tau concentrations between the first and second dive, comparing subjects who breathed air to subjects who breathed oxygen after the first dive. Oxygen breathing did not influence obtained NfL or tau values. No significant differences were found for these proteins at any point. At 30-45 min after diving there were no differences in GFAp results between divers breathing oxygen or air, but results for GFAp were higher 120 min after diving when breathing air compared to oxygen. Results for analyses of the effect of oxygen breathing on GFAp, NfL and tau are presented in Table 1 . 


\section{Effect of prior diving ( $48 \mathrm{~h}$ before sample 4)}

The difference between samples obtained before each dive (samples 1 and 4) was compared separately for subjects breathing oxygen and air after their first dive, to see if a residual effect was present. No significant differences in GFAp, NfL and tau concentrations immediately before the two dives were found, suggesting that neither diving nor oxygen breathing $48 \mathrm{~h}$ prior to the second dive had an effect on the results obtained. The results are presented in Table 2.

\section{Effect of diving}

GFAp, NfL and tau blood levels after diving were analysed linearly, comparing changes between sample 1 and samples $2-4$ as well as between samples 4 and samples 5-6 in sequence (Table 3), and also according to breathing gas used, ignoring order of the dives (Table 4).

\section{GFAp}

When divers were analysed together irrespective of postdive breathing gas, GFAp concentrations were significantly decreased at 30-45 min after the first dive but not at $120 \mathrm{~min}$ post-dive. No significant changes in GFAp concentrations were observed after the second dive (Table 3 ). In addition, there were no significant changes in GFAp after either dive when analysed based on post-dive breathing gas (Table 4).

\section{NfL}

Variations in mean NfL concentrations observed after the two dives did not reach statistical significance (Tables 3 and 4).

\section{Tau}

Mean protein tau concentrations increased after each dive. When all divers were analysed together, irrespective of

Table 1 Differences in GFAp, NfL and tau values after breathing oxygen compared to air-adjusted for period effect

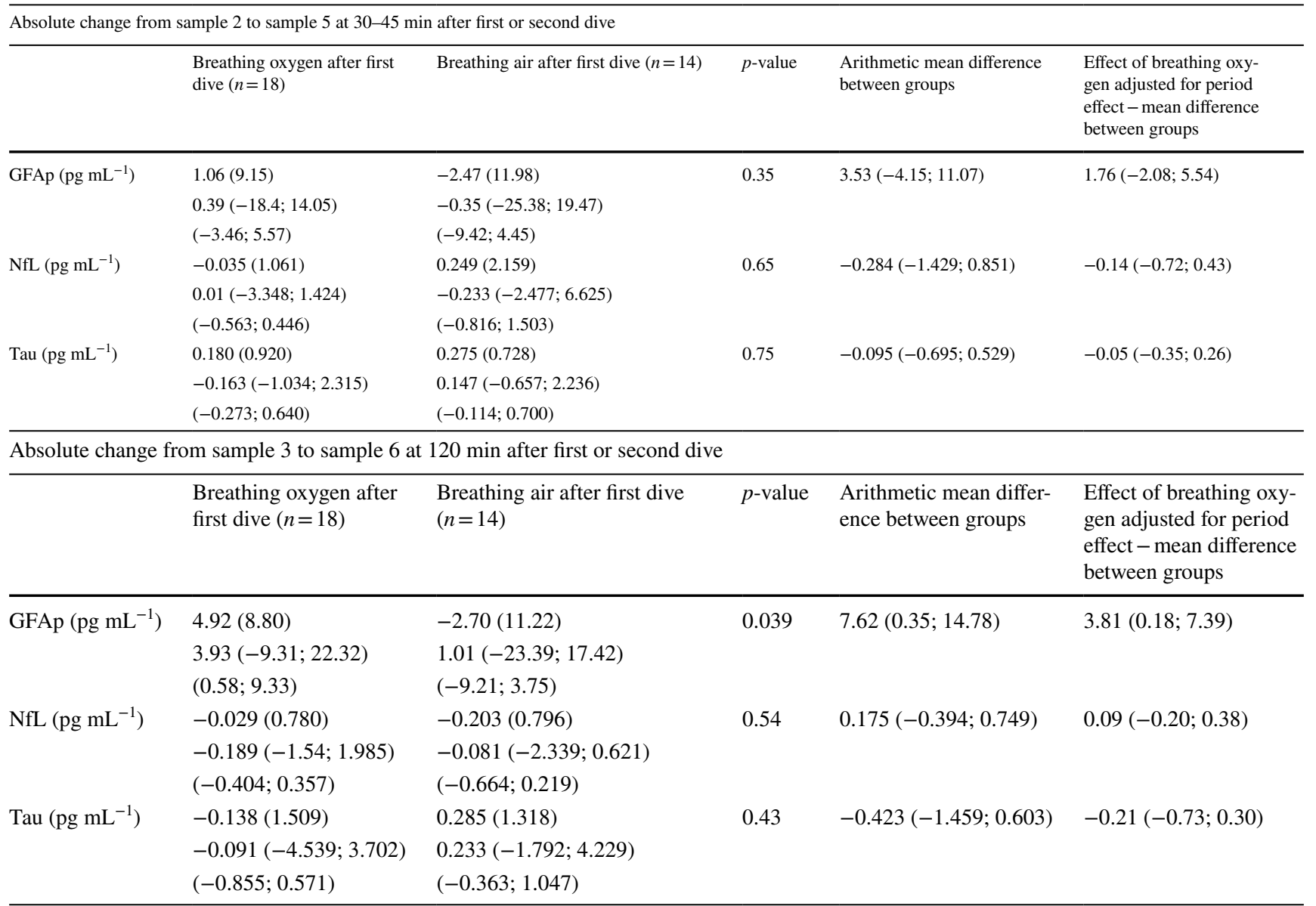

Mean (standard deviation)/median (range)/(95\% CI for mean using the inversion of Fisher's non-parametric permutation test) are presented. For comparison between groups the Fisher's non-parametric permutation test was used. Mean difference between groups is presented with a $95 \%$ confidence interval. The arithmetic mean difference between groups equals twice the effect of given treatment (oxygen or air) 
Table 2 Differences in GFAp, NfL and tau values before each dive-estimation of carryover effect

\begin{tabular}{|c|c|c|c|c|c|c|}
\hline \multicolumn{7}{|c|}{ Absolute difference between samples 1 and 4} \\
\hline & $\begin{array}{l}\text { Subjects breathing oxygen } \\
\text { after first dive }(n=18)\end{array}$ & $\begin{array}{l}p \text {-value within } \\
\text { group }\end{array}$ & $\begin{array}{l}\text { Subjects breathing air } \\
\text { after first dive }(n=14)\end{array}$ & $\begin{array}{l}p \text {-value within } \\
\text { group }\end{array}$ & $\begin{array}{l}\text { Mean absolute differ- } \\
\text { ence between groups }\end{array}$ & $\begin{array}{l}p \text {-value } \\
\text { between } \\
\text { groups }\end{array}$ \\
\hline \multirow{3}{*}{$\begin{array}{l}\text { GFAp } \\
\left(\mathrm{pg} \mathrm{mL}^{-1}\right)\end{array}$} & $-0.04(12.77)$ & 0.99 & $-6.88(15.38)$ & 0.12 & $6.83(-3.14 ; 17.12)$ & 0.18 \\
\hline & $-4.52(-20.05 ; 27.82)$ & & $-8.36(-33.76 ; 15.47)$ & & & \\
\hline & $(-6.29 ; 6.39)$ & & $(-15.90 ; 2.06)$ & & & \\
\hline \multirow{3}{*}{$\begin{array}{l}\mathrm{NfL} \\
\quad\left(\mathrm{pg} \mathrm{mL} \mathrm{L}^{-1}\right)\end{array}$} & $0.02(1.16)$ & 0.95 & $0.60(2.81)$ & 0.62 & $-0.58(-1.97 ; 0.80)$ & 0.52 \\
\hline & $-0.28(-1.56 ; 3.47)$ & & $-0.22(-1.33 ; 9.62)$ & & & \\
\hline & $(-0.520 ; 0.599)$ & & $(-0.65 ; 2.21)$ & & & \\
\hline \multirow{3}{*}{$\begin{array}{l}\text { Tau } \\
\left(\mathrm{pg} \mathrm{mL}^{-1}\right)\end{array}$} & $-0.04(0.52)$ & 0.75 & $0.01(0.48)$ & 0.96 & $-0.05(-0.42 ; 0.31)$ & 0.78 \\
\hline & $-0.08(-1.36 ; 0.86)$ & & $0.16(-0.96 ; 0.56)$ & & & \\
\hline & $(-0.30 ; 0.21)$ & & $(-0.27 ; 0.29)$ & & & \\
\hline
\end{tabular}

Mean (standard deviation)/median (range)/(95\% CI for mean using the inversion of Fisher's non-parametric permutation test) is presented. For comparison within groups Fisher's non-parametric permutation test for matched pairs was used. The confidence interval for the mean difference between groups was based on Fishers non-parametric permutation test

breathing gas used after a certain dive, the changes were statistically significant 120 min after both dives, and at 30-45 min after the second dive. Tau concentrations decreased between the dives with mean tau concentrations obtained prior to both dives being almost identical (Table 3). When changes in tau were analysed based on breathing gas used, mean levels were significantly increased at 30-45 min following oxygen breathing and at 120 min regardless of breathing gas used (Table 4).

Mean tau had increased by $29.1 \%$ (SD $44.7 \%$ ) and 33.9\% (SD 81.7\%) at $120 \mathrm{~min}$ after the first and second dive, respectively. When all 64 dives were analysed together, the mean tau increase at $120 \mathrm{~min}$ after diving was $31.5 \%$ (SD $66.4 \%$ ). In one diver, tau increased by $428 \%$ after the second dive. If this outlier value was omitted, the tau increase after the second dive was $21.2 \%$ (SD $41.5 \%$ ), and was $25.2 \%$ (SD $43.7 \%$ ) at $120 \mathrm{~min}$ for the remaining 63 dives taken together.

\section{Correlation between markers of neuronal injury and venous gas emboli}

Absolute values for GFAp, NfL and tau concentrations at 30-45 and $120 \mathrm{~min}$ after each dive as well as changes in GFAp, NfL and tau concentrations at these points were tested for correlation with matching $\mathrm{VGE}_{\text {max }}$, and $\mathrm{KISS}_{30 \min }$ or KISS ${ }_{120 \min }$ values, respectively. No correlations were found. Results are presented in Appendices 1 and 2 (Figs. 1, $2,3)$.

\section{Discussion/conclusions}

The present study found that tau levels in blood increased after a dive made to an equivalent of $42 \mathrm{msw}$ depth. When an identical dive was performed 2 days later, the increases in tau observed after the first dive were reproduced. Tau changes seemed to be fast, with a measurable increase as soon as 30-45 min after diving, with yet higher tau levels found after $120 \mathrm{~min}$. The design of the present study did not allow us to determine peak tau levels, or determine when they appeared, but after $48 \mathrm{~h}$ tau blood levels had returned to values obtained before the first dive.

Breathing normobaric oxygen after diving did not affect the tau blood levels obtained. Hence, tau changes could be analysed without regard to the breathing gas used after each dive. Nevertheless, the results were strengthened by the fact that increases in tau concentrations seen $120 \mathrm{~min}$ after diving also remained statistically significant for both groups when dives with oxygen and air breathing afterwards were analysed separately. There was no correlation between absolute tau concentrations or their changes and VGE loads, which indicates that tau release is not affected by the presence of VGE.

Tau blood levels rise after brain damage and neuronal cell death, with median concentrations of $49.5 \mathrm{pg} \mathrm{mL}^{-1}$ observed at $48 \mathrm{~h}$ after cardiac arrest among patients with poor neurological outcome (Mattson 2017), whereas in the present study, tau concentrations were $2.2 \mathrm{pg} \mathrm{mL}^{-1} 120 \mathrm{~min}$ after diving. The physiological mechanism that causes tau levels to increase, albeit to a lesser degree, after presumed neuronal stress without manifest injury to the CNS has not been identified. Breathing normobaric oxygen after diving 
Table 3 Changes in GFAp, NfL and tau after diving

\begin{tabular}{|c|c|c|c|}
\hline Sample 1: before first dive & Absolute value $(n=32)$ & $\begin{array}{l}\text { Absolute change compared to sample } 1 \\
(n=32)\end{array}$ & $p$-value \\
\hline GFAp $\left(\mathrm{pg} \mathrm{mL}^{-1}\right)$ & $\begin{array}{l}60.3(26.2) \\
51.9(31.2 ; 139.2)\end{array}$ & & \\
\hline $\mathrm{NfL}\left(\mathrm{pg} \mathrm{mL}^{-1}\right)$ & $\begin{array}{l}7.84(7.72) \\
6.12(3.4 ; 47.79)\end{array}$ & & \\
\hline Tau $\left(\mathrm{pg} \mathrm{mL}^{-1}\right)$ & $\begin{array}{l}1.70(0.89) \\
1.5(0.4 ; 4.3)\end{array}$ & & \\
\hline Sample 2: 30-45 min after first dive & Absolute value $(n=32)$ & $\begin{array}{l}\text { Absolute change compared to sample } 1 \\
(n=32)\end{array}$ & $p$-value \\
\hline GFAp $\left(\mathrm{pg} \mathrm{mL}^{-1}\right)$ & $\begin{array}{l}56.6(24.0) \\
49.4(29 ; 139)\end{array}$ & $\begin{array}{l}-3.69(10.08) \\
-2.90(-30.21 ; 20.22) \\
(-7.29 ;-0.06)\end{array}$ & 0.045 \\
\hline $\mathrm{NfL}\left(\mathrm{pg} \mathrm{mL} \mathrm{L}^{-1}\right)$ & $\begin{array}{l}7.75(8.28) \\
5.66(3.21 ; 51.01)\end{array}$ & $\begin{array}{l}-0.09(0.92) \\
-0.24(-1.29 ; 3.22) \\
(-0.40 ; 0.24)\end{array}$ & 0.61 \\
\hline Tau $\left(p g \mathrm{~mL}^{-1}\right)$ & $\begin{array}{l}1.89(0.84) \\
1.86(0.39 ; 3.68)\end{array}$ & $\begin{array}{l}0.18(0.73) \\
0.20(-2.08 ; 2.22) \\
(-0.08 ; 0.44)\end{array}$ & 0.17 \\
\hline Sample 3: $120 \mathrm{~min}$ after first dive & Absolute value $(n=32)$ & $\begin{array}{l}\text { Absolute change compared to sample } 1 \\
(n=32)\end{array}$ & $p$-value \\
\hline GFAp $\left(p g \mathrm{~mL}^{-1}\right)$ & $\begin{array}{l}56.5(20.9) \\
55.4(26.5 ; 128.5\end{array}$ & $\begin{array}{l}-3.82(12.88) \\
-3.31(-40.28 ; 15.25) \\
(-8.52 ; 0.87)\end{array}$ & 0.11 \\
\hline $\mathrm{NfL}\left(\mathrm{pg} \mathrm{mL} \mathrm{L}^{-1}\right)$ & $\begin{array}{l}8.11(8.33) \\
6.4(3.05 ; 51.61)\end{array}$ & $\begin{array}{l}0.271(1.11) \\
0.11(-1.68 ; 3.83) \\
(-0.13 ; 0.67)\end{array}$ & 0.18 \\
\hline Tau $\left(\mathrm{pg} \mathrm{mL}^{-1}\right)$ & $\begin{array}{l}2.18(1.47) \\
1.84(0.45 ; 8.13)\end{array}$ & $\begin{array}{l}0.48(1.03) \\
0.27(-1.03 ; 5.21) \\
(0.15 ; 0.82)\end{array}$ & 0.0008 \\
\hline $\begin{array}{l}\text { Sample 4: before second dive ( } 48 \mathrm{~h} \text { after the } \\
\text { first dive) }\end{array}$ & Absolute value $(n=32)$ & $\begin{array}{l}\text { Absolute change compared to sample } 1 \\
(n=32)\end{array}$ & $p$-value \\
\hline GFAp $\left(\mathrm{pg} \mathrm{mL}^{-1}\right)$ & $\begin{array}{l}57.3(20.6) \\
58.3(33 ; 135.7)\end{array}$ & $\begin{array}{l}-3.03(14.16) \\
-5.83(-33.76 ; 27.82) \\
(-8.18 ; 2.14)\end{array}$ & 0.24 \\
\hline $\mathrm{NfL}\left(\mathrm{pg} \mathrm{mL}^{-1}\right)$ & $\begin{array}{l}8.11(8.43) \\
6.02(3.6 ; 51.25)\end{array}$ & $\begin{array}{l}0.27(2.03) \\
-0.28(-1.56 ; 9.62) \\
(-0.38 ; 0.97)\end{array}$ & 0.55 \\
\hline Tau $\left(p g \mathrm{~mL}^{-1}\right)$ & $\begin{array}{l}1.68(0.81) \\
1.54(0.58 ; 3.16)\end{array}$ & $\begin{array}{l}-0.02(0.50) \\
0.01(-1.36 ; 0.86) \\
(-0.20 ; 0.16)\end{array}$ & 0.81 \\
\hline Sample 5: $30-45 \mathrm{~min}$ after second dive & Absolute value $(n=32)$ & $\begin{array}{l}\text { Absolute change compared to sample } 4 \\
(n=32)\end{array}$ & $p$-value \\
\hline GFAp $\left(p g \mathrm{~mL}^{-1}\right)$ & $\begin{array}{l}56.2(19.5) \\
55(31.5 ; 127.6)\end{array}$ & $\begin{array}{l}-1.14(8.34) \\
-0.88(-14.61 ; 13.69) \\
(-4.21 ; 1.91)\end{array}$ & 0.45 \\
\hline $\mathrm{NfL}\left(\mathrm{pg} \mathrm{mL}{ }^{-1}\right)$ & $\begin{array}{l}7.84(7.64) \\
6.16(3.44 ; 47.66)\end{array}$ & $\begin{array}{l}-0.27(2.07) \\
-0.11(-7.54 ; 6.38) \\
(-1.01 ; 0.45)\end{array}$ & 0.48 \\
\hline
\end{tabular}


Table 3 (continued)

\begin{tabular}{|c|c|c|c|}
\hline Sample 5: 30-45 min after second dive & Absolute value $(n=32)$ & $\begin{array}{l}\text { Absolute change compared to sample } 4 \\
(n=32)\end{array}$ & $p$-value \\
\hline Tau $\left(\mathrm{pg} \mathrm{mL}^{-1}\right)$ & $\begin{array}{l}2.11(1.32) \\
1.89(0.53 ; 5.27)\end{array}$ & $\begin{array}{l}0.42(0.92) \\
0.24(-1.67 ; 3.09) \\
(0.10 ; 0.76)\end{array}$ & 0.0098 \\
\hline Sample 6: $120 \mathrm{~min}$ after second dive & Absolute value $(n=32)$ & $\begin{array}{l}\text { Absolute change compared to sample } 4 \\
(n=32)\end{array}$ & $p$-value \\
\hline GFAp $\left(\mathrm{pg} \mathrm{mL}^{-1}\right)$ & $\begin{array}{l}58.1(20.3) \\
57.3(30.5 ; 138)\end{array}$ & $\begin{array}{l}0.80(10.06) \\
0.95(-18.24 ; 22.63) \\
(-2.81 ; 4.39)\end{array}$ & 0.65 \\
\hline $\mathrm{NfL}\left(\mathrm{pg} \mathrm{mL} \mathrm{L}^{-1}\right)$ & $\begin{array}{l}8.01(8.38) \\
6.12(3.05 ; 51.95)\end{array}$ & $\begin{array}{l}-0.10(1.49) \\
0.19(-7.31 ; 1.94) \\
(-0.61 ; 0.34)\end{array}$ & 0.84 \\
\hline Tau $\left(\mathrm{pg} \mathrm{mL}^{-1}\right)$ & $\begin{array}{l}2.23(1.56) \\
1.96(0.6 ; 6.74)\end{array}$ & $\begin{array}{l}0.54(1.20) \\
0.12(-0.90 ; 4.91) \\
(0.14 ; 0.97)\end{array}$ & 0.0041 \\
\hline
\end{tabular}

Mean (SD)/median (min; max)/(95\% CI for mean using the inversion of Fisher's non-parametric permutation test) is presented. For comparison within groups the Fisher's non-parametric permutation test for matched pairs was used

did not affect tau blood levels in the present study, but it is still possible that exposure to supranormal partial pressures of oxygen might affect tau levels. Other possible causes of increased tau blood levels are increased ambient pressure per se, changes in cerebral perfusion during immersion, or compression stress. As there was no correlation between VGE and tau levels, decompression stress seems unlikely to have influenced the results.

Trimix breathing gas is used for deep dives and contains oxygen, helium and nitrogen. Tau increased by $98.8 \%$ in a small pilot study where ten divers performed repeated deep dives between 52-90 msw over 4 days using trimix (Rosén et al. 2019), with an oxygen partial pressure of $130 \mathrm{kPa}$ during the dive and up to $160 \mathrm{kPa}$ during decompression; nitrogen pressures at depth were around 176-193 $\mathrm{kPa}$. In the present study the partial pressures of oxygen and nitrogen at depth were $109 \mathrm{kPa}$ and $406 \mathrm{kPa}$, respectively. The trimix study found no correlation between VGE loads and tau concentrations, either in terms of their absolute values or their changes. The larger relative increase in tau blood levels after diving in the trimix study compared to the present study could be due to differences in dive depths, oxygen partial pressures, breathing gas, and number of dives between the two studies.

In another study, when submariners were exposed to a pressure equivalent to $30 \mathrm{msw}(401 \mathrm{kPa})$ for $36 \mathrm{~h}$ in a dry hyperbaric chamber, then followed by slow decompression over a further $70 \mathrm{~h}$, no significant change in tau blood concentration was seen (Rosén et al. 2020). The submariners experienced a lower ambient pressure than the divers in the present study, but their duration of exposure was longer.
Tau was sampled before exposure, at 33-34 h of exposure, and after exposure had ended. No samples were obtained at either 30-45 or 120 min of exposure, making comparison between the present study and the submariner study difficult; it is possible that tau levels may have increased after initial pressurization to $30 \mathrm{msw}(401 \mathrm{kPa})$ and then decreased, reaching baseline levels before a sample was obtained at 33-34 h. In the submariner study, the maximum oxygen partial pressure was $50 \mathrm{kPa}$, with a maximum nitrogen partial pressure of about $350 \mathrm{kPa}$, and the rate of decompression was $0.375-0.5 \mathrm{msw} / \mathrm{h}$; this rate is much slower than that of the present study ( $9 \mathrm{msw} / \mathrm{min})$.

It could be speculated that the changes in tau seen after diving were caused by oxidative stress and increased levels of ROS but the fact that tau increases were unaffected by normobaric oxygen breathing during 30 min after diving, an exposure three times longer and just slightly less hyperoxic than the dive itself, makes such a mechanism less plausible. Higher nitrogen partial pressures in this study did not coincide with larger tau increases, compared to the trimix study.

Different preconditioning techniques have been shown to decrease VGE load after diving though none of them were employed in this study, which makes it impossible to judge their potential effect on tau concentrations. Oxygen breathing before diving would probably have reduced VGE load but at the same time caused oxidative stress to the diver. As there was no association between VGE and tau, and the effect of oxidative stress on tau is uncertain, it is questionable if oxygen breathing before diving would have affected the results. Possible effects of other preconditioning 
Table 4 Changes in GFAp, NfL and tau—breathing air or oxygen after diving

\begin{tabular}{|c|c|c|}
\hline \multicolumn{3}{|c|}{ Breathing oxygen after diving } \\
\hline 30-45 min after diving & Absolute change $(n=32)$ & $p$-value \\
\hline \multirow[t]{3}{*}{ GFAP $\left(\mathrm{pg} \mathrm{mL}^{-1}\right)$} & $-1.76(9.72)$ & 0.31 \\
\hline & $-1.26(-22.73 ; 13.69)$ & \\
\hline & $(-5.24 ; 1.75)$ & \\
\hline \multirow[t]{3}{*}{$\mathrm{NfL}\left(\mathrm{pg} \mathrm{mL} \mathrm{L}^{-1}\right)$} & $-0.24(2.014)$ & 0.52 \\
\hline & $-0.28(-7.54 ; 6.38)$ & \\
\hline & $(-0.95 ; 0.452)$ & \\
\hline \multirow[t]{3}{*}{ Tau $\left(\mathrm{pg} \mathrm{mL}^{-1}\right)$} & $0.30(0.77)$ & 0.03 \\
\hline & $0.22(-2.08 ; 2.22)$ & \\
\hline & $(0.02 ; 0.57)$ & \\
\hline 120 min after diving & Absolute change $(n=32)$ & $p$-value \\
\hline \multirow[t]{3}{*}{ GFAP $\left(\mathrm{pg} \mathrm{mL}^{-1}\right)$} & $-1.99(10.65)$ & 0.31 \\
\hline & $-2.43(-34.37 ; 15.25)$ & \\
\hline & $(-5.81 ; 1.83)$ & \\
\hline \multirow[t]{3}{*}{$\mathrm{NfL}\left(\mathrm{pg} \mathrm{mL}^{-1}\right)$} & $-0.08(1.637)$ & 0.83 \\
\hline & $0.08(-7.31 ; 3.83)$ & \\
\hline & $(-0.64 ; 0.45)$ & \\
\hline \multirow[t]{3}{*}{ Tau $\left(\mathrm{pg} \mathrm{mL}^{-1}\right)$} & $0.60(1.29)$ & 0.0014 \\
\hline & $0.24(-1.03 ; 5.21)$ & \\
\hline & $(0.16 ; 1.05)$ & \\
\hline \multicolumn{3}{|l|}{$30-45$ min after diving } \\
\hline \multirow[t]{3}{*}{ GFAP $\left(\mathrm{pg} \mathrm{mL}^{-1}\right)$} & $-3.07(8.89)$ & 0.06 \\
\hline & $-2.41(-30.21 ; 20.22)$ & \\
\hline & $(-6.27 ; 0.11)$ & \\
\hline \multirow[t]{3}{*}{$\mathrm{NfL}\left(\mathrm{pg} \cdot \mathrm{mL}^{-1}\right)$} & $-0.12(1.03)$ & 0.53 \\
\hline & $-0.01(-3.59 ; 1.83)$ & \\
\hline & $(-0.49 ; 0.25)$ & \\
\hline \multirow[t]{3}{*}{ Tau $\left(\mathrm{pg} \cdot \mathrm{mL}^{-1}\right)$} & $0.307(0.903)$ & 0.06 \\
\hline & $0.20(-1.67 ; 3.09)$ & \\
\hline & $(-0.01 ; 0.63)$ & \\
\hline $120 \mathrm{~min}$ after diving & Absolute change $(n=32)$ & $p$-value \\
\hline \multirow[t]{3}{*}{ GFAP $\left(\mathrm{pg} \mathrm{mL}^{-1}\right)$} & $-1.02(12.82)$ & 0.66 \\
\hline & $0.04(-40.28 ; 22.63)$ & \\
\hline & $(-5.68 ; 3.59)$ & \\
\hline \multirow[t]{3}{*}{$\mathrm{NfL}\left(\mathrm{pg} \mathrm{mL}^{-1}\right)$} & $0.24(0.89)$ & 0.12 \\
\hline & $0.27(-1.27 ; 1.94)$ & \\
\hline & $(-0.07 ; 0.56)$ & \\
\hline \multirow[t]{3}{*}{ Tau $\left(\mathrm{pg} \mathrm{mL}^{-1}\right)$} & $0.42(0.90)$ & 0.0034 \\
\hline & $0.21(-0.90 ; 4.18)$ & \\
\hline & $(0.12 ; 0.74)$ & \\
\hline
\end{tabular}

For continuous variables mean (SD)/median (min; max)/(95\% CI for mean using the inversion of Fisher's non-parametric permutation test) are presented. For comparison within groups the Fisher's non-parametric permutation test for matched pairs was used

techniques such as sauna-induced heat exposure or wholebody vibration remain to be elucidated.

A difference in GFAp blood concentrations 120 min after diving was observed when samples from divers breathing oxygen were compared to their paired air samples, which suggests that oxygen might have influenced the change in GFAp blood levels obtained at this point. Therefore, it was not prudent to analyse changes in GFAp blood levels without regard to breathing gas used after the dive. However, when GFAp samples were analysed in two separate groups based on breathing gas, GFAp blood levels were not significantly changed at any point, though a 
decrease seen in GFAp 30-45 min after dives followed by air breathing almost reached significance $(p=0.06)$. When all dives were analysed irrespective of breathing gas postdive, GFAp was significantly decreased at 30-45 min after

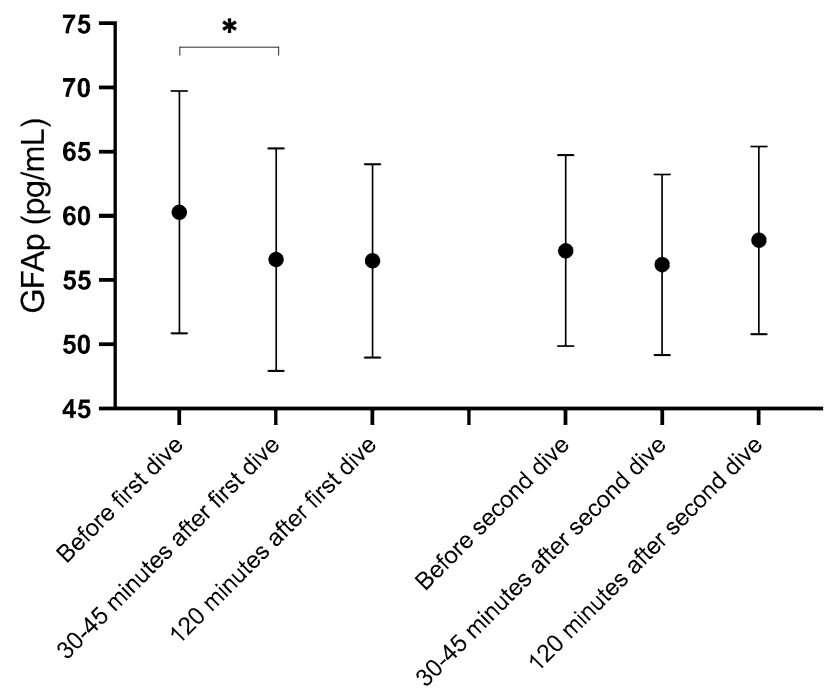

Fig. 1 GFAp concentrations after diving. Mean GFAp concentrations with $95 \%$ confidence intervals

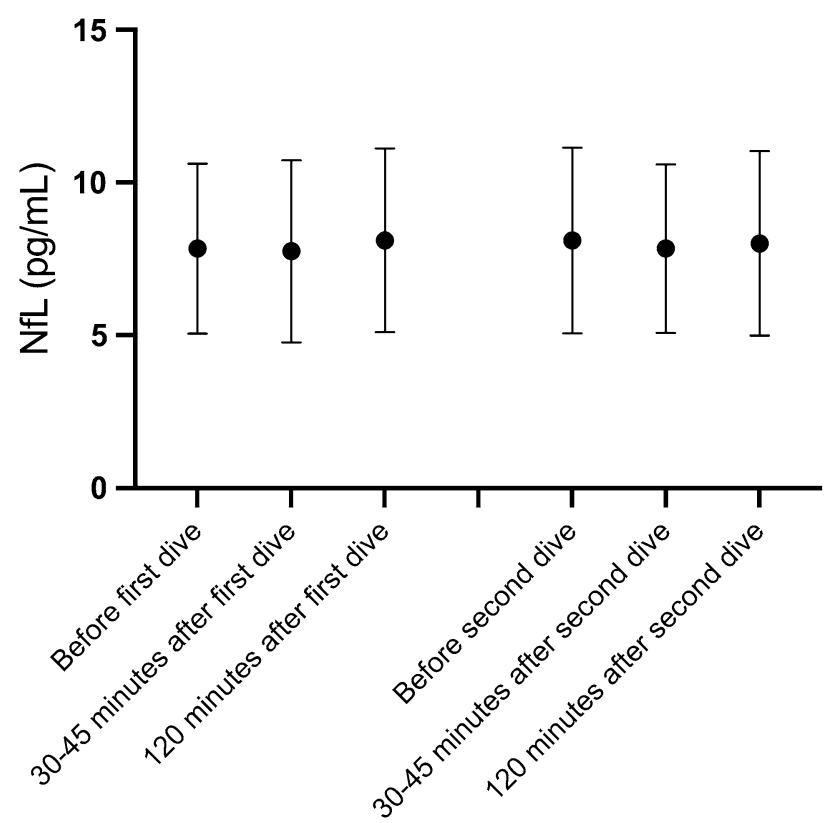

Fig. $2 \mathrm{NfL}$ concentrations after diving. Mean NfL concentrations with $95 \%$ confidence intervals

the first dive but not at the same point after the second. There were no significant changes at $120 \mathrm{~min}$ after either dive. These disparate results regarding changes in GFAp blood levels after diving suggest that it would not be useful as a marker of dive-related, presumably neuronal, stress. There was no correlation between GFAp concentrations, or their changes and VGE loads.

NfL levels in blood did not change significantly in the

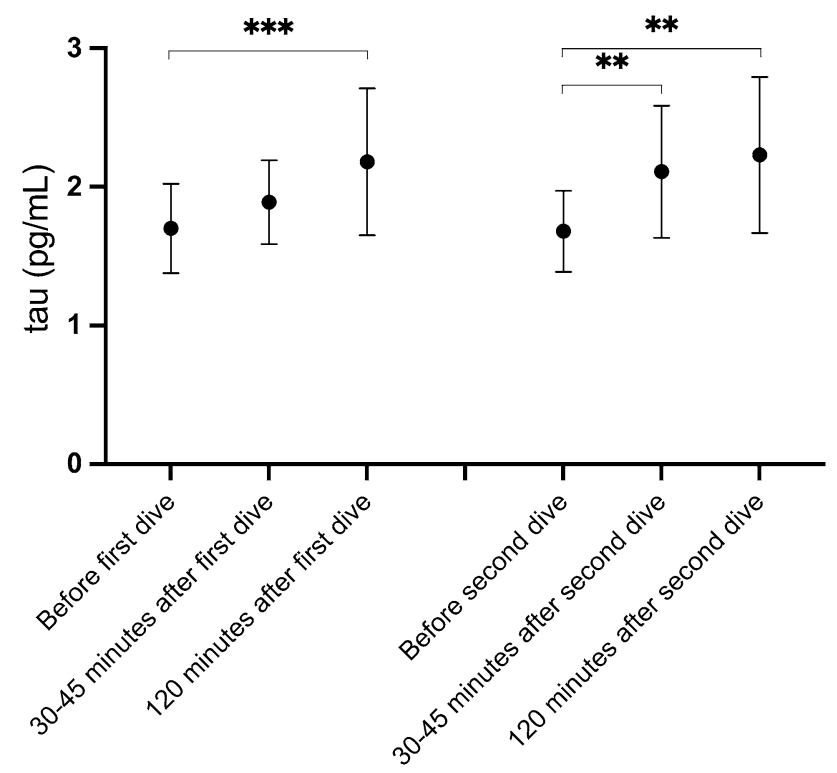

Fig. 3 Protein tau concentrations after diving. Mean tau concentrations with $95 \%$ confidence intervals

present study, which is consistent with the aforementioned studies on deep repeated diving and prolonged hyperbaric exposure of submariners. The results do not support neuroaxonal injury, although the slow dynamics of $\mathrm{NfL}$, with maximum increases seen later than seven days after an insult (Shahim et al. 2016), makes it a less well-suited marker in the setting of this study. NfL levels in blood were not influenced by oxygen breathing after diving or correlated to VGE load.

Dehydration is common after diving and could cause increased concentrations of proteins measured in blood but this parameter was not assessed, which is a shortcoming of this study. However, as tau increased while NfL remained unchanged and GFAp either remained unchanged or decreased, significant dehydration seems unlikely. Active hydration before diving is a preconditioning technique (Gempp et al. 2008) which theoretically may cause a decrease in venous protein concentrations after diving, but it was not employed in the present study.

Samples were taken only up to $120 \mathrm{~min}$ after each dive. Continued sampling may have yielded more detailed data on changes in tau concentration after diving, potentially making it possible to determine maximum tau values in blood after each dive. 
In this study, tau blood levels increased after diving. The use of repeated, uniform dive exposures is a strength of the study and the fact that tau increases were similar after the first and the second dives makes the results convincing. Tau concentrations in blood were not correlated to VGE. Based on these results, as well as the aforementioned pilot study on deep trimix diving, tau seems to be a promising marker of dive-related, presumably neuronal, stress.

A larger study where subjects perform repeated dives to different depths and durations is necessary both to validate these results and to establish if there is a quantitative relationship between dive exposure and tau levels in blood. Blood sampling should ideally be frequent and continued for at least hours after each dive.

Supplementary Information The online version contains supplementary material available at https://doi.org/10.1007/s00421-022-04892-9.

Acknowledgements The authors would like to thank Statistiska Konsultgruppen, Gothenburg, Sweden (statistical analyses), the Swedish Navy, the Swedish Society for Medical Military Officers, Dr. Lesley Blogg of SLB Consulting (Doppler ultrasound measurements), participating individuals and research personnel for their contributions to this study.

Author contributions The project was conceived and designed by AR, NO and MG. AR, AK and NO collected the blood samples. HZ and JS were responsible for blood analyses. AR drafted the manuscript, which NO, AK, MG, GS, HSL, JS and HZ critically revised. All authors approved the final version before submission.

Funding Open access funding provided by University of Gothenburg. The Swedish society for military medical officers provided financial contribution to this study. HZ is a Wallenberg Scholar supported by grants from the Swedish Research Council (\#2018-02532), the European Research Council (\#681712), Swedish State Support for Clinical Research (\#ALFGBG-720931), the Alzheimer Drug Discovery Foundation (ADDF), USA (\#201809-2016862), and the UK Dementia Research Institute at UCL.

\section{Declarations}

Conflict of interest $\mathrm{HZ}$ has served at scientific advisory boards for Denali, Roche Diagnostics, Wave, Samumed, Siemens Healthineers, Pinteon Therapeutics and $\operatorname{CogRx}$, has given lectures in symposia sponsored by Fujirebio, Alzecure and Biogen, and is a co-founder of Brain Biomarker Solutions in Gothenburg AB (BBS), which is a part of the GU Ventures Incubator Program (outside submitted work).

Availability of data and material The datasets generated and analysed during the current study are available from the corresponding author on reasonable request.

Ethics approval The study was approved by Swedish ethical review authorities as EPN Dnr 352-14 with supplementary approvals T84715, T1032-18 and 202-05525.

Consent to participate Written consent to participate was obtained from all study subjects.
Consent for publication All authors have approved publication of the manuscript.

Open Access This article is licensed under a Creative Commons Attribution 4.0 International License, which permits use, sharing, adaptation, distribution and reproduction in any medium or format, as long as you give appropriate credit to the original author(s) and the source, provide a link to the Creative Commons licence, and indicate if changes were made. The images or other third party material in this article are included in the article's Creative Commons licence, unless indicated otherwise in a credit line to the material. If material is not included in the article's Creative Commons licence and your intended use is not permitted by statutory regulation or exceeds the permitted use, you will need to obtain permission directly from the copyright holder. To view a copy of this licence, visit http://creativecommons.org/licenses/by/4.0/.

\section{References}

Balestra C, Germonpré P (2016) Correlation between patent foramen ovale, cerebral "lesions" and neuropsychometric testing in experienced sport divers: does diving damage the brain? Front Psychol 7:696 (PMID 27242609)

Bast-Pettersen R, Skare Ø, Nordby K-C, Skogstad M (2015) A twelveyear longditudinal study of neuropsychological function in nonsaturation professional divers. Int Arch Occup Environ Haelth 88(6):669-682 (PMID 25355537)

Bergman J, Dring A, Zetterberg H et al (2016) Neurofilament light in CSF and serum is a sensitive marker for axonal white matter injury in MS. Neurol Neuroimmunol Neuroinflamm 3(5):e271 (PMID 27536708)

Bhullar J, Bhopale VM, Yang M, Sethuraman K, Thom SR (2016) Microparticle formation by platelets exposed to high gas pressures-an oxidative stress response. Free Radic Biol Med 101:154-162 (PMID 27751909)

Billaut F, Gueit P, Faure S, Costalat G, Lemaitre F (2018) Do elite breath-hold divers suffer from mild-short term memory impairments? Appl Physiol Nutr Metab 43(3):247-251 (PMID 29053942)

Bittermann N (2004) CNS oxygen toxicity. Undersea Hyperb Med 31(1):63-72 (PMID 15233161)

Blatteau JE, Pontier JM (2009) Effect of in-water recompression with oxygen to $6 \mathrm{msw}$ versus normobaric oxygen breathing on bubble formation in divers. Eur J Appl Physiol 106:691-695 (PMID 19424716)

Blogg SL, Gennser M, Møllerløkken A, Brubakk AO (2014) Ultrasound detection of vascular decompression bubbles: the influence of new technology and considerations on bubble load. Diving Hyperb Med 44:35-44 (PMID 24687484)

Bosco G, Yang Z-J, di Tano G et al (2010) Effect of in-water oxygen prebreathing at different depths on decompression-induced bubble formation and platelet activation. J Appl Physiol 108:10771083 (PMID 20185629)

Bosco G, Rizzato A, Quartesan S et al (2018) Spirometry and oxidative stress after rebreather diving in warm water. Undersea Hyperb Med 45(2):191-198 (PMID 29734571)

Brenner M (2014) Role of GFAP in CNS injuries. Neurosci Lett 565:7-13 (PMID 24508671)

Camporesi EM, Bosco G (2014) Mechanisms of action of hyperbaric oxygen therapy. Undersea Hyperb Med 41(3):247-252 (PMID 24984320) 
Castagna O, Gempp E, Blatteau JE (2009) Pre-dive normobaric oxygen reduces bubble formation in SCUBA divers. Eur J Appl Physiol 106:167-172 (PMID 19219451)

Clarke JE (2015) Moving in extreme environments: inert gas narcosis and underwater activities. Extrem Physiol Med 4:1 (PMID 25713701)

Coco M, Buscemi A, Perciavalle V et al (2019) Cognitive deficits and white matter alterations in highly trained scuba divers. Fron Psychol 10:2376 (PMID 31695655)

Cordes P, Keil R, Bartsch T et al (2000) Neurologic outcome of compressed-air diving. Neurology 55:1743-1746 (PMID 11113237)

di Battista AP, Moes KA, Shiu MY et al (2018) High intensity interval training is associated with alterations in blood biomarkers related to brain injury. Front Physiol 9:1367 (PMID 30323770)

Eckenhoff RG, Olstad CS, Carrod G (1990) Human dose-response relationship for decompression and endogenous bubble formation. J Appl Physiol 69:914-918 (PMID 2246178)

Eftedal OS, Lydersen S, Brubakk AO (2007) The relationship between gas bubbles and adverse effects of decompression after air dives. Undersea Hyperb Med 34(2):99-105 (PMID 17520861)

Ergen M, Uslu A, Caglar O et al (2017) Evaluation of cognitive performance in professional divers by menas of event-related potentials and neuropsychology. Clin Neurophysiol 128(4):579-588 (PMID 28231476)

Evered L, Silbert B, Scott DA, Zetterberg H, Blennow K (2018) Association of changes in plasma neurofilament light and tau levels with anesthesia and surgery. JAMA Neurol 75(5):542-547 (PMID 29459944)

Femenia T, Gimenez-Cassina A, Codeluppi S et al (2018) Disrupted neuroglial metabolic coupling after peripheral surgery. J Neurosci 38(2):452-464 (PMID 29175959)

Ferrer MD, Sureda A, Batle JM et al (2007) Scuba diving enhances endogenous antioxidant defences in lymphocytes and neutrophils. Free Radic Res 41(3):274-281 (PMID 1736495)

Foerch C, Niessner M, Back T et al (2012) Diagnostic accuracy of plasma glial fibrillary acidic protein for differentiating intracerebral haemorrhage and cerebral ischemia in patients with symptoms of acute stroke. Clin Chem 58(1):237-245 (PMID 22125303)

Gempp E, Blatteau JE, Pontier J-M, Balestra C, Louge P (2008) Preventive effect of pre-dive hydration on bubble formation in divers. Br J Sports Med 43:224-228 (PMID 18308884)

Gempp E, Blatteau JE (2010) Preconditioning methods and mechanisms for preventing the risk of decompression sickness in scuba divers: a review. Res Sports Med 18(3):205-218 (PMID 20623437)

Germonpre P, Pontier J-M, Gempp E, Blatteau J-E, Deneweth S, Lafére P, Marroni A, Balestra C (2010) Pre-dive vibration effect on bubble formation after a 30-m dive requiring a decompression stop. Aviat Space Environ Med 81:1044-1048 (PMID 20027852)

Germonpré P, Balestra C (2017) Preconditioning to reduce decompression stress in scuba divers. Aerosp Med Hum Perform 88(2):114 120 (PMID 28095955)

Gill J, Latour L, Diaz-Arrastia R et al (2018) Glial fibrillary acidic protein elevations relate to neuroimaging abnormalities after mild TBI. Neurology 91(15):e1385-e1389 (PMID 30209234)

Gren M, Shahim P, Lautner R, Wilson DH, Andreasson U et al (2016) Blood biomarkers indicate mild neuroaxonal injury and increased amyloid beta production after transient hypoxia during breathhold diving. Brain Inj 30:1226-1230 (PMID 27389622)

Grønning M, Aarli JA (2011) Neurological effects of deep diving. J Neurol Sci 304(1-2):17-21 (PMID 21377169)

Hemelryck W, Germonpre P, Papadopoulou V, Rozloznik M, Balestra C (2014) Long term effects of recreational SCUBA diving on higher cognitive function. Scand J Med Sci Sports 24:928-934 (PMID 23902533)

Jankowski LW, Tikuisis P, Nishi RY (2004) Exercise effects during diving and decompression on postdive venous gas emboli. Aviat Space Environ Med 75(6):489-495 (PMID 15198273)

Kahlil M, Teunissen CE, Otto M, Piehl F, Sormani MP et al (2018) Neurofilaments as biomarkers in neurological disorders. Neurology 14:577-589 (PMID 30171200)

Kisman KE, Masurel G, Guillerm R (1978a) Bubble evaluation code for Doppler ultrasonic decompression data. Undersea Biomed Res 5(1):28

Kisman KE, Masurel G, Lagrue D, LePerchon JC (1978b) Evaluation de la qualité d'une decompression basée sur la detection ultrasonore de bulles. Med Aéro Spat Med Sub Hyp 67:293-297

Knauth M, Ries S, Pohimann S et al (1997) Cohort study of multiple brain lesions in sport divers: role of a patent foramen ovale. BMJ 314:701-705 (PMID 9116544)

Kohshi K, Tamaki H, Lemaitre F et al (2014) Brain damage in commercial breath-hold divers. PLoS ONE 9(8):e105006 (PMID 25115903)

Mattson N, Zetterberg H, Nielsen N, Blennow K, Dankiewicz J et al (2017) Serum tau and neurological outcome in cardiac arrest. Ann Neurol 82:665-675 (PMID 28981963)

Neselius S, Brisby H, Theodorsson A, Blennow K, Zetterberg H, Marcusson J (2012) CSF-biomarkers in Olympic boxing: diagnosis and effects of repetitive head trauma. PLoS ONE 7(4):e33606 (PMID 22496755)

Nishi RY, Kisman KE, Eatock BC, Buckingham IP, Masurel G (1981) Assessment of decompression profiles and divers by Doppler ultrasonic monitoring. Proceedings of 7th Symposium underwater physiology, Athens. UMS Inc., Bethesda MD, pp 717-727

Papadopoulou V, Germonpré P, Cosgrove D et al (2018) Variability in circulating gas emboli after a same scuba dive exposure. Eur Appl Physiol 118(6):1255-1264 (PMID 29616324)

Rosén A, Oscarsson N, Kvarnström A et al (2019) Serum tau concentration after diving - an observational pilot study. Diving Hyperb Med 49(2):88-95 (PMID 31177514)

Rosén A, Gennser M, Oscarsson N et al (2020) Biomarkers of neuronal damage in saturation diving - a controlled observational study. Eur J Appl Physiol 120(12):2773-2784 (PMID 32975632)

Sawatzky KD (1991) The relationship between intravascular Doppler detected gas bubbles and decompression sickness after bounce diving in humans. In: Master of science thesis. York University, Toronto, Canada. ISBN 0-315-70405-5

Sato C, Barthélemy NR, Mawuenyega KG et al (2018) Tau kinetics in neurons and the human nervous system. Neuron 97(6):1284-1298. e7 (PMID 29566794)

Shahim P, Arnell P, Kvarnström A et al (2015) Cerebrospinal fluid markers of central nervous system injury in decompression illness - a case-controlled pilot study. Diving Hyperb Med 45:240 243 (PMID 26687311)

Shahim P, Gren M, Liman V et al (2016) Serum neurofilament light protein predicts clinical outcome in traumatic brain injury. Sci Rep 6:36791 (PMID 27819296)

Shahim P, Tegner Y, Marklund N, Blennow K, Zetterberg H (2018) Neurofilament light and tau as blood biomarkers for sports-related concussion. Neurology 90(20):e1780-e1788 (PMID 29653990)

Siracusa R, Fusco R, Cuzzocrea S (2019) Astrocytes: role and functions in brain pathologies. Front Pharmacol 10:1114 (PMID 31611796)

Slosman DO, de Ribauspierre S, Chicherio C et al (2004) Negative effects of frequency, depth and environment in recreational scuba diving: the Geneva "memory dive" study. Br J Sports Med 38(2):108-114 (PMID 15039241)

Taylor CL, Macdiarmid JI, Ross JAS et al (2006) Objective neuropsychological test performance of professional divers reporting a 
subjective complaint of "forgetfulness or loss of concentration." Scand J Work Environ Health 32(4):310-317 (PMID 16932829)

Tetzlaff K, Friege L, Hutzelmann A et al (1999) Magnetic resonance signal abnormalities and neuropsychological deficits in elderly compressed-air divers. Eur Neurol 42(4):194-199 (PMID 10567814)

Todnem K, Nyland H, Kambestad BK, Aarli JA (1990) Influence of occupational diving upon the nervous system: an epidemiological study. Br J Ind Med 47(10):708-714 (PMID 2171631)

Todnem K, Nyland H, Skeidsvoll H et al (1991) Neurological long term consequences of deep diving. Br J Ind Med 48:258-266 (PMID 2025592)

Vaernes RJ, Kløve H, Ellertsen B (1989) Neuropsychologic effects of saturation diving. Undersea Biomed Res 16(3):233-251 (PMID 2741256)
Wang YF, Hatton GI (2009) Astrocytic plasticity and patterned oxycontin neuronal activity: dynamic interactions. J Neurosci 29(6):1743-1754 (PMID 19211881)

Zetterberg H, Hietala A, Jonsson M et al (2006) Neurochemical aftermath of amateur boxing. Arch Neurol 63:1277-1280 (PMID 16966505)

Zetterberg H, Blennow K (2016) Fluid biomarkers for mild traumatic brain injury and related conditions. Nat Rev Neurol 12:563-574 (PMID 27632903)

Publisher's Note Springer Nature remains neutral with regard to jurisdictional claims in published maps and institutional affiliations. 